\title{
STOCHASTIC PROCESSES CONNECTED WITH HARMONIC FUNCTIONS
}

\author{
BY \\ JOANNE ELLIOTT(1) AND WILLIAM FELLER
}

0 . Introduction. The family of transformations

$$
T_{t} f(x)=\pi^{-1} \int_{-\infty}^{+\infty} \frac{t}{t^{2}+(x-y)^{2}} f(y) d y, \quad t>0
$$

define a semi-group of bounded linear transformations from $C[-\infty,+\infty]$ to itself. The salient feature of this semi-group is that the function

$$
u(t, x)=T_{t} f(x)
$$

is harmonic in the half-plane $t>0$. The infinitesimal generator of this semigroup is of the form

$$
\Omega F(x)=\pi^{-1} P \cdot \int_{-\infty}^{+\infty} \frac{F^{\prime}(y)}{y-x} d y,
$$

the integral being taken in the sense of a Cauchy principal value. The precise definition of this generator is found in Theorem 3.3 as a byproduct of the present investigation $\left({ }^{2}\right)$.

In [2] the first of the present authors studied a class of semi-groups from $C[-a, a]$ to itself with $0<a<\infty$, whose infinitesimal generator $\Omega_{a}$ is obtained from $(0.2)$ by truncation:

$$
\Omega_{a} F(x)=\pi^{-1} P \cdot \int_{-a}^{+a} \frac{F^{\prime}(y)}{y-x} d y
$$

the exact definition of this operator is found in Definition 1.1 of the present paper. These semi-groups share with $(0.1)$ the property of being positivity preserving and norm-not-increasing. Among them there is a minimal semigroup $T_{t}^{(a)}$ characterized by the boundary conditions:

$$
T_{t}^{(a)} f( \pm a)=0 . \quad f \in C[-a,+a] .
$$

Although the present paper in no way depends on the consideration of

Received by the editors November 21, 1955.

(1) Research sponsored by the Office of Ordnance Research under Contract Number DA-19059-ORD-1611 at Mount Holyoke College.

(2) Hille [6] has investigated $(0.1)$ as a semi-group from $L_{p}(-\infty,+\infty)$ to itself when $p>1$ and found the infinitesimal generator. These semi-groups on $C[-\infty,+\infty]$ and $L(-\infty,+\infty)$ apparently have not been investigated previously. 
function spaces, it is now desirable to give the probabilistic background of our semi-groups.

To (0.1) there corresponds probabilistically the Cauchy process, a separable Markovian process with stationary transition probabilities,

$$
P(t, x, S)=\pi^{-1} \int_{S} \frac{t}{t^{2}+(x-y)^{2}} d y
$$

where $S$ stands for a Borel set. It is easily seen that the path functions $X(t)$ of this process are discontinuous. In fact, if $S$ is an interval not containing $x$ then (0.5) implies that as $\Delta t \downarrow 0$,

$$
(\Delta t)^{-1} \operatorname{Pr}\{X(t+\Delta t) \in S \mid X(t)=x\}=\pi^{-1} \int_{S} \frac{d y}{(y-x)^{2}}+0(1) .
$$

This relation determines the probability distribution of the saltuses of $X(t)$ and characterizes the Cauchy process.

In like manner the semi-group determined by $(0.3)-(0.4)$ depends on an integral transformation, whose $\operatorname{kernel}\left({ }^{3}\right) P_{a}(t, x, S)$ defines the transition probabilities of a new stochastic process with path functions confined to the interval $(-a, a)$. One of the purposes of the present paper is to investigate the connection between this process and the Cauchy process.

$\mathrm{M}$. Kac [7] was the first to study the absorbing barrier process connected with the Cauchy process: this is the process confined to $(-a, a)$ whose path functions coincide with those of a Cauchy process as long as $|X(t)|<a$, but stop being defined from the first moment when $|X(t)| \geqq a$. Kac showed how to calculate the probability $F_{a}(t, x)$ that the lifetime of this process will exceed $t$ when the starting point is $x$. For the Cauchy process itself $1-F_{a}(t, x)$ is the probability distribution of the first passage time from $x$ into the set $|s|>a$.

The investigation in [2] was inspired partly by Kac's result. The fact that Kac was led to the operator $(0.3)$ combined with the minimality of the semigroup determined by the boundary condition (0.4) suggests that $P_{a}(t, x, S)$, represents the transition probabilities of the absorbing barrier process. If this is true one should have

$$
F_{a}(t, x)=P_{a}(t, x, E), \quad E=(-a, a) .
$$

Purely formally this equation can be written in the form

$$
F_{a}=T_{t}^{a}
$$

where 1 is the unit function. Since $\Omega_{a}$ is the infinitesimal generator of $T_{t}^{(a)}$, this means that at least in some operational sense, $F_{a}(t, X)$ is the solution of the functional equation

(3) The Laplace transform (1.6) of $P_{a}(t, x, S)$ is described in $\$ 1$. 


$$
\frac{\partial}{\partial t} F_{a}(t, x)-\Omega_{a} F_{a}(t, x)=0\left(^{4}\right)
$$

with the lateral conditions

$$
F_{a}(t, \pm a)=0, \quad F_{a}(0, x)=1 .
$$

The Laplace transforms of these equations are valid and are given at the end of $\S 1$.

We now come to the contents of the present paper. If $P_{a}(t, x, S)$ is indeed the transition probability of the absorbing barrier process we can draw several conclusions.

(a) Concerning first passage times. The quantity $F_{a}(t, x)-F_{a}(t+h, x)$ represents the probability in the Cauchy process of a path starting at $x \in(-a, a)$ to stay within $(-a, a)$ for a time $t$ and then with the time interval $(t, t+h)$ to leave $(-a, a)$. The position at time $t$ has, by assumption, the probability distribution $P_{a}(t, x, S)$, and the probability of a saltus leading out of $(-a, a)$ is governed by $(0.6)$. Combining these results and letting $h \rightarrow 0$ one is led to expect that $\left.{ }^{5}\right)$

$$
\begin{aligned}
-\frac{\partial}{\partial t} F_{a}(t, x) & =\pi^{-1} \int_{-a}^{a} P_{a}(t, x, d y) \int_{|z|>a} \frac{d z}{(z-y)^{2}} \\
& =2 a \pi^{-1} \int_{-a}^{a} P_{a}(t, x, d y)\left(a^{2}-y^{2}\right)^{-1} .
\end{aligned}
$$

The Laplace transform version of this equation is contained in (1.15).

Now this equation is curious and contains an apparent contradiction. For, it states that $-\partial F_{a}(t, x) / \partial t$ is the transform, under the semi-group $T_{t}^{(a)}$, of the unbounded function $\phi(y)=2 \pi^{-1}\left(a^{2}-y^{2}\right)^{-1}$. From the general behavior of the semi-group for bounded functions one should accordingly expect that

$$
-\frac{\partial}{\partial t} F_{a}(t, x) \rightarrow 2 \pi^{-1}\left(a^{2}-x^{2}\right)^{-1},
$$$$
t \rightarrow 0 .
$$

This, however, leads to immediate contradictions. It has been proved in [3] that actually (0.10) holds (at least in the Laplace transform sense) with

(4) Kac's fundamental equation (8.12) may be interpreted as the statement that $F_{a}$ is a "weak" solution of $(0.8)$ in an appropriate Hilbert space. The uniqueness theorem proved by Kac shows that his boundary conditions are essentially equivalent to (0.9). It in no way contradicts the existence of the other semi-groups and processes generated by $\Omega_{a}$. It was the study of Kac's equation (8.12) that led to the analysis of $\Omega_{a}$ in [2].

(5) Equation (0.10) presupposes that with probability one the jump occurs from a point in the interior of $(-a, a)$ to a point $z$ with $|z|>a$. That this is so follows indirectly from the validity of (0.10), but has been proved previously by McKean [9]. A similar statement is true for all stable processes with index $\alpha \leqq 1$. For $\alpha>1$ the equation corresponding to $(0.10)$ contains an additional term which accounts for the possibility that $z= \pm a$ is the first point outside $(-a, a)$ actually to be reached. 


$$
-\frac{\partial}{\partial t} F_{a}(t, x) \rightarrow 0, \quad t \rightarrow 0
$$

and not (0.11).

This result shows that the extension of the semi-group from bounded to unbounded functions is by no means obvious. The corresponding extension of the resolvent and of $\Omega_{a}$ was studied in [3] and reveals surprising analytical features. The definition of the extended operator corresponding to $\Omega_{a}$ is found in Definition 1.3.

(b) Relations between transition probabilities. The kernel $P_{a}(t, x, S)$ defines, for each $x \in(-a, a)$ and each $t>0$, a measure on the Borel subsets of $(-a, a)$. We find it convenient to extend $P_{a}$ to a measure on $(-\infty, \infty)$ by stipulating that $P_{a}(t, x, \cdot)$ ascribes measure 0 to Borel sets contained in the complement of $(-a, a)$.

For the Cauchy process, a passage from a point $x \in(-a, a)$ at time $t$ to a set $S \subset(-a, a)$ can occur in two ways: either during the entire time interval $(0, t)$, the path is constrained to $(-a, a)$, or a first passage into the exterior of $(-a, a)$ occurs at some time $s<t$. Using the argument which led to (0.11), we conclude that we should have

$$
P(t, x, S)=P_{a}(t, x, S)+\int_{n}^{t} d s \int_{-a}^{a} P_{a}(s, x, d y) \int_{|z|>a} \frac{P(t-s, z, S)}{\pi(z-y)^{2}} d z
$$

provided $x \in(-a, a)$ and $S \subset(-\infty, \infty)$.

By the same argument and under the same conditions, we should have for $a<b,|x|<a$, and $S \subset(-\infty, \infty)$,

$$
P_{b}(t, x, S)=P_{a}(t, x, S)
$$

$$
+\int_{0}^{t} d s \int_{-a}^{a} P_{a}(s, x, d y) \int_{a<|z|<b} \frac{P_{b}(t-s, z, S)}{\pi(z-y)^{2}} d z .
$$

We shall not attempt to derive equations $(0.13)$ and $(0.14)$ directly for the absorbing barrier processes. Instead, we start from the kernel $P_{a}$, using the fact that it describes a Markov process in $(-a, a)$. In Theorems 3.1 and 2.1 we prove the relations corresponding to $(0.13)$ and $(0.14)$ for the Laplace transforms of the densities of $P_{a}, P$, and $P_{b}$, and in Corollaries 3.1 and 2.1 we show that (0.13) and (0.14) actually hold for our kernels.

With these relations at hand, we can now check that the kernel $P_{a}$ gives indeed the transition probabilities for the absorbing barrier process of the Cauchy process. For arbitrary $x$, denote by $n_{x}$ the integer such that $\left(2 n_{x}-1\right) a$ $\leqq x<\left(2 n_{x}+1\right) a$, and by $I_{x}$ the interval $\left(\left(2 n_{x}-1\right) a,\left(2 n_{x}+1\right) a\right)$. Then put

$$
P_{p e r}(t, x, S)=P_{a}\left(t, x-2 n_{x} a, S-2 n_{x} a\right) .
$$

Probabilistically, $P_{p e r}$ describes, for fixed $I_{x}$, a replica of the $P_{a}$ process re- 
stricted to the interval $I_{x}$. In the sequel, we shall refer to this as the $P_{a}$ process in $I_{x}$.

Since $P(t, x, S)=P(t, x-2 n a, S-2 n a)$, it is easily seen that if $(0.13)$ holds, then

$$
\begin{aligned}
P(t, x, S)= & P_{p e r}(t, x, S) \\
& +\int_{0}^{t} d s \int_{-\infty}^{\infty} P_{p e r}(s, x, d y) \int_{z \bigoplus_{I_{x}}} \frac{P(t-s, z, S)}{\pi(z-y)^{2}} d z,
\end{aligned}
$$

for $x \in I_{x}$.

A standard iteration procedure applied to $(0.16)$ gives

$$
P(t, x, S)=\sum_{k=0}^{n} P_{p e r}^{(k)}(t, x, S)+r_{n}(t, x, S),
$$

where the terms in the sum are defined recursively by

$$
\begin{aligned}
P_{p e r}^{(0)}(t, x, S) & =P_{\text {per }}(t, x, S), \\
P_{\text {per }}^{(k+1)}(t, x, S) & =\int_{0}^{t} d s \int_{-\infty}^{\infty} P_{\text {per }}^{(k)}(s, x, d y) \int_{z \notin I_{x}} \frac{P_{\text {per }}(t-s, z, S)}{\pi(z-y)^{2}} d z .
\end{aligned}
$$

Now define,

$$
P_{p e r}^{*}(t, x, S)=\sum_{k=0}^{\infty} P_{p e r}^{(k)}(t, x, S) .
$$

The series converges since all the terms in $(0.17)$ are positive and $P(t, x, S)$ $\leqq 1$.

We next show that $P$ coincides with $P^{*}$ for almost all $x$. For each $t, x$, and $S$ the sequence $\left\{r_{n}\right\}$ is a decreasing, positive sequence. From Theorem $4.1,(7.1)-(7.4)$, and Theorem 7.1, it follows that if $f$ is continuous in $[-a, a]$ and periodic $2 \mathrm{a}$, then for $x \in I_{x}$

$$
\int_{-\infty}^{\infty} P(t, x, d y) f(y)=\int_{-\infty}^{\infty} P_{p e r}^{*}(t, x, d y) f(y) .
$$

In particular if $f(y) \equiv 1$, we must have

$$
\lim _{n \rightarrow \infty} \int_{-\infty}^{\infty} r_{n}(t, x, d y)=0 .
$$

Hence $\lim _{n \rightarrow \infty} r_{n}(t, x, S)=0$ and $P(t, x, S)=P_{p e r}^{*}(t, x, S)$ for all $x \in(-\infty, \infty)$, except the odd multiples of $\pm a$.

Probabilistically, $P_{p e r}^{*}$ gives the transition probabilities of a Markov process consisting of an infinite succession of $P_{a}$ processes, such that whenever one terminates at $y$ a new initial position $z$ is chosen according to (0.6), and 
the process starts afresh in the interval $I_{z}$. It is clear that $P_{a}$ gives the transition probabilities for the absorbing barrier process in $(-a, a)$ for the $P_{\text {per }}^{*}$ process, and hence also for the Cauchy process in view of the preceding paragraph.

(c) Return processes. In [2] there was constructed a whole family of semigroups generated by $\Omega_{a}$, and each yields the transition probabilities of a stochastic process. Among these, the simplest to describe are the return processes $\left({ }^{6}\right)$. They are formally and probabilistically analogous to corresponding processes in diffusion theory. Now, in diffusion the path functions are continuous, and the infinitesimal generator is a differential operator. It is therefore a priori to be expected that all processes with the same local behavior should be governed by the same infinitesimal generator. No such reasoning applies in the case of the Cauchy process. And in fact, we shall show that in the latter case, there exist more general return processes than those considered in [5]; and the infinitesimal generator does not necessarily coincide with $\Omega_{a}$.

The general return process considered here may be described as follows. Consider a particle moving in the interval $(-a, a)$ according to the transition probabilities $P(t, x, S)$ of the Cauchy process as long as the particle remains within $(-a, a)$. When it for the first time leaves the interval, it jumps to a point $z$ with $|z|>a$. We now associate with each point $|z|>a$ a probability $\tau(z)(0 \leqq \tau(z) \leqq 1)$, that the particle is returned to a random point $Y \in(-a, a)$ with a given probability distribution $\operatorname{Pr}\{Y \in S\}=p(z, S)$. The process then continues as before from the point $Y$ to which it has been returned.

The special case where

$$
p(z, S)= \begin{cases}p_{1}(S) & \text { for } z>a, \\ p_{2}(S) & \text { for } z<a\end{cases}
$$

has been treated in [2].

The argument which led to (0.13) now leads to an integral equation for the transition probabilities $\mathfrak{B}_{a}$ of this return process. The Laplace transform of this integral equation is (4.2). It is shown in $\$ 4$ that to arbitrarily prescribed $p$ and $\tau$ the integral equation has a solution which, in a certain sense, is minimal. This solution determines a semi-group from $C[-a, a]$ to itself and, accordingly, a stochastic process. These are discussed in $\$ 5$. An example in $\$ 4$ shows that our integral equation may have several probabilistically admissible solutions. Also, even when $\tau(z) \equiv 1$ one may have a norm-decreasing semi-

${ }^{(6)}$ All other processes can be obtained by a passage to the limit analogous to that described in [5]. We have thus a one-one correspondence between the processes in [2] and the diffusion processes constructed in [4]. The interpretation given in [5] applies in both cases. However, a probabilistic investigation of the behavior of the path functions of the processes formally analogous to the elastic and reflecting barrier processes of diffusion theory remains an open problem. 
group, with $\mathfrak{B}_{a}(t, x, E)<1\left({ }^{7}\right)$. Equation (4.4) gives a sufficient condition for this not to occur. It will be seen that the infinitesimal generators of our return processes coincide with $\Omega_{a}$ only if $(0.22)$ holds.

(d) Connection with harmonic functions. Consider the semi-group (0.1) applied only to continuous functions which are odd and periodic with period 2a. Obviously such a function $f$ can be prescribed arbitrarily in $(0, a)$ and we are dealing therefore in effect with a semi-group from $C[0, a]$ to itself. Again $T_{t} f$ is harmonic, odd and periodic, and therefore admits of a Fourier sine series. In fact if $f \sim \sum_{n=1}^{\infty} a_{n} \sin (n \pi / a) x$ is the formal Fourier series of $f$, then

$$
T_{t} f=\sum_{n=1}^{\infty} e^{-(n x / a) t} \sin \frac{n \pi}{a} x .
$$

Here $T_{t} f$ is a function harmonic in $0<x<a, t>0$, uniquely determined by the boundary conditions $T_{t} f(0)=T_{t} f(a)=0$.

Now these are the boundary conditions of the absorbing barrier process on $(0, a)$ and one might suspect that $(0.23)$ represents the semi-group corresponding to the absorbing barrier process. This conjecture may appear strengthened by the fact that in the case of the homogeneous diffusion (governed by the parabolic equation $u_{t}=u_{x x}$ ) the above construction does indeed lead to the absorbing barrier process, and that the latter admits of a Fourier representation of the form $(0.23)$ (with the exponent replaced by $\left.n^{2} \pi^{2} t / a^{2}\right)$.

Actually (0.23) is a transition semi-group and determines a Markovian process, but it is not the absorbing barrier process. Its infinitesimal generator is of the form (7.28) with tan replaced by cot. It is not clear whether and how the process is related to the Cauchy process.

More generally, the class of functions $u$ harmonic in the semi-strip $\alpha_{1} \leqq x \leqq \alpha_{2}$, $t>0$, and subject to the classical boundary conditions

$$
p_{i} u\left(\alpha_{i}, t\right)+(-1)^{i} q_{i} \frac{\partial}{\partial x} u\left(\alpha_{i}, t\right)=0
$$

defines a transition semi-group from $C\left[\alpha_{1}, \alpha_{2}\right]$ to itself, and therefore a Markovian process in $\left[\alpha_{1}, \alpha_{2}\right]$, provided $p_{i} \geqq 0, q_{i} \geqq 0$. The semi-group (0.23) corresponds to $q_{1}=q_{2}=0$. The corresponding cosine series,

$$
T_{t} f=\sum_{n=0}^{\infty} a_{n} e^{-(n \pi / a) \iota} \cos \frac{n \pi}{a} x
$$

(7) The probabilistic explanation of this curious phenomenon is as follows: The probability distribution $p(z, S)$ may make it probable that each jump out of the interval $E$ will bring the path closer to a boundary point $\pm a$; if $T_{n}$ are the time distances between successive jumps out of $E$, then it may occur that the expectation of $\sum T_{n}$ is finite, so that infinitely many jumps out of $E$ may occur in a finite time. When this occurs the path function of our return process is, of course, no longer determined. This phenomenon is well-known in the theory of Markov processes in denumerable spaces and has been discussed by Doob [1]. 
where $f \sim \sum_{n=0}^{\infty} a_{n} \cos (n \pi / a) x$, represents a transition semi-group from $C[0, a]$ to itself such that $u(x, t)=T_{t} f(x)$ is a harmonic function satisfying the boundary conditions $(0.24)$ with $p_{1}=p_{2}=0$. The formal analogue for the heat equation is the reflecting barrier solution; it satisfies the same boundary conditions and has a representation of the form (0.25) (with $n \pi / a$ in the exponent replaced by $\left.n^{2} \pi^{2} / a^{2}\right)$. An alternative representation for the semi-group $(0.25)$ is obtained by extending the definition of $f(x)$ from $(0, a)$ to all $x$ in such a way that $f$ becomes even and periodic with period 2a. With this convention $(0.1)$ is equivalent to $(0.25)$. The semi-groups associated with the other boundary conditions admit of representations analogous to $(0.25)$ and $(0.23)$ by means of trigonometric series.

The boundary conditions (0.24) are not the most general ones assuring positivity. For example, the boundary condition $u(a, t)=u(-a, t)$ for the interval $(-a, a)$ leads to the semi-group

$$
T_{t} f(x)=\sum_{n=0}^{\infty} e^{-(n \pi / a) t}\left\{a_{n} \cos \frac{n \pi}{a} x+b_{n} \sin \frac{n \pi}{a} x\right\},
$$

which is a transition semi-group.

It is not clear that any of these semi-groups should have a more intimate connection with the Cauchy process. However, in \$7, we show that both $(0.25)$ and $(0.26)$ can be interpreted as return processes. The corresponding return probabilities are $\tau(z) \equiv 1$ and $p(z, S)$ given by (7.1) and (7.23), respectively.

1. Preliminaries. In this section we collect some results to which we shall refer frequently in the sequel.

DEFINITION 1.1. If (i) $F \in C[-a,+a]$ is absolutely continuous on $(-a$, $+a)$, (ii) $\left[F^{\prime}(x)\right]^{2}\left(a^{2}-x^{2}\right)^{1 / 2} \in L(-a,+a)$, and (iii) for some $h \in C[-a,+a]$,

$$
\pi^{-1} P . \int_{-a}^{a} \frac{F^{\prime}(t)}{t-x} d t=h(x)
$$

almost everywhere on $[-a,+a]$, then we define $\Omega_{a} F=h$ as a linear transformation from $C[-a,+a]$ to itself.

TheOREM 1.1. For each $\lambda>0$, the equation

$$
\lambda F_{a}-\Omega_{a} F_{a}=f
$$

with $f \in C[-a,+a]$ has a unique solution $F_{a} \in C[-a,+a]$ under the boundary condition

$$
F_{a}(-a)=F_{a}(a)=0 .
$$

This solution is of the form

$$
F_{a}(x)=\int_{-a}^{a} \Gamma_{a}(x, y ; \lambda) f(y) d y
$$


where $\Gamma_{a}(x, y ; \lambda) \geqq 0$ and is symmetric in $x$ and $y$. The function $F_{a}$ has a continuous derivative in $(-a,+a)$.

Proof. The proof of these statements may be found in [2]. Note that $F_{a}(x)$ depends on $\lambda$. We shall find it convenient to omit this dependence in writing, however. The kernel $\Gamma_{a}(x, y ; \lambda)$ has the following connection with the absorbing barrier transition probabilities described in the first part of the Introduction. We define

$$
\gamma_{a}(x, S ; \lambda)=\int_{S} \Gamma_{a}(x, y ; \lambda) d y
$$

with $S$ a Borel subset of $[-a,+a]$. Then if $P_{a}(t, x, S)$ denotes, as before, the transition probability for the absorbing barrier process, we have

$$
\gamma_{a}(x, S ; \lambda)=\int_{0}^{\infty} e^{-\lambda t} P_{a}(t, x, S) d t .
$$

As we pointed out in the Introduction, it will be necessary to consider $\Gamma_{a}$ operating on certain unbounded functions. This class of functions is described as follows:

Definition 1.2. Let $U[-a,+a]$ denote the Banach space of functions $f$ of the form

$$
f(x)=\phi(x)\left(a^{2}-x^{2}\right)^{-1}
$$

where $\phi \in C[-a,+a]$ and $\|f\|_{U}=\|\phi\|_{C}$.

In $[3, \S 3]$, we showed that the transformation $f \rightarrow F_{a}$ defined by (1.4) is a bounded linear transformation from $U[-a,+a]$ to itself or to $C[-a,+a]$.

If $f \in U[-a,+a]$, then $F_{a}$ as defined by (1.4) does not necessarily satisfy (1.2). In [3, Theorem 4.1] we derived the functional equation analogous to (1.2) which is satisfied in the more general situation. These results are summed up in the following:

Definition 1.3. We define

$$
\left(a^{2}-x^{2}\right) \tilde{\Omega}_{a} F(x)=\pi^{-1} P \cdot \int_{-a}^{a} \frac{F^{\prime}(t)\left(a^{2}-t^{2}\right)}{t-x} d t-\pi^{-1} \int_{-a}^{a} F(t) d t
$$

for those $F \in C[-a,+a]$ which are absolutely continuous on any proper subinterval of $(-a,+a)$ with $\left[F^{\prime}(x)\right]^{2}\left(a^{2}-x^{2}\right)^{3 / 2} \in L(-a,+a)$ and for which the right-hand side of (1.8) is almost everywhere equal to an element of $C[-a,+a]$.

We then have

THEOREM 1.2. If $F_{a}$ is defined by (1.4) with $f \in U[-a,+a]$ for some $\lambda>0$, then $F_{a}$ is the unique solution in $C[-a,+a]$ of

$$
\lambda F_{a}-\tilde{\Omega}_{a} F_{a}=f \text {. }
$$


Proof. See [3, Theorem 4.1].

We now go on to the Laplace transform version of the discussion in section (a) of the Introduction.

THEOREM 1.3. The equation

$$
\lambda u-\Omega_{a} u=0,
$$$$
\lambda>0,
$$

has exactly two linearly independent solutions in $C[-a,+a]$ given by

$$
\xi_{a}^{ \pm}(x)=\pi^{-1} \int_{-a}^{a} \frac{\Gamma_{a}(x, y ; \lambda)}{a \pm y} d y
$$

which satisfy

$$
\xi_{a}^{+}(a)=\overline{\xi_{a}}(-a)=0, \quad, \xi_{a}^{+}(-a)=\overline{\xi_{a}}(a)=1 .
$$

Proof. See [3, Theorem 3.1].

In the language of $\S(\mathrm{a})$ of the Introduction, $\xi_{a}^{+}$and $\xi_{a}^{-}$are the Laplace transforms of the right and left escape probability densities, respectively.

We shall usually make use of $\xi_{a}^{+}+\xi_{\bar{a}}=\xi_{a}$. Interpreted according to (0.10),

$$
\begin{aligned}
\xi_{a}(x) & =-\int_{0}^{\infty} e^{-\lambda t} \frac{\partial}{\partial t} F_{a}(t, x) d t \\
& =2 a \pi^{-1} \int_{-a}^{a} \frac{\Gamma_{a}(x, y ; \lambda)}{a^{2}-y^{2}} d y .
\end{aligned}
$$

On the other hand, if we integrate by parts

(1.14) $\xi_{a}(x)=F_{a}(0, x)-\lambda \int_{0}^{\infty} e^{-\lambda t} F_{a}(t, x) d t=1-\lambda \int_{-a}^{a} \Gamma_{a}(x, y ; \lambda) d y$, using (0.7) and (0.9). We should thus have the relation

$$
\xi_{a}(x)=1-\lambda \int_{-a}^{a} \Gamma_{a}(x, y ; \lambda) d y=2 a \pi^{-1} \int_{-a}^{a} \frac{\Gamma_{a}(x, y ; \lambda)}{a^{2}-y^{2}} d y .
$$

Formula (1.15) is proved analytically in [3, Theorem 3.1].

2. The identity between kernels. This section contains the Laplace transform version of (0.13) and (0.14) namely,

Theorem 2.1. If $\Gamma_{a}$ and $\Gamma_{b}$ are defined as in Theorem 1.1 with $a<b$, then

$$
\begin{aligned}
\Gamma_{a}(x, y ; \lambda)= & \Gamma_{b}(x, y ; \lambda) \\
& -\pi^{-1} \int_{-a}^{a} \Gamma_{a}(x, u ; \lambda)\left\{\int_{a<|z|<b} \frac{\Gamma_{b}(z, y ; \lambda)}{(z-u)^{2}} d z\right\} d u
\end{aligned}
$$

when $|x|<a,|y|<a$ and 


$$
\Gamma_{b}(x, y ; \lambda)=\pi^{-1} \int_{-a}^{a} \Gamma_{a}(x, u ; \lambda)\left\{\int_{a<|z|<b} \frac{\Gamma_{b}(z, y ; \lambda)}{(z-u)^{2}} d z\right\} d u
$$

when $|x|<a, a<|y|<b$.

Proof. We can reduce (2.1) and (2.2) to a single relation by setting $\Gamma_{a}(x, y ; \lambda)=0$ for $|x|>a,|y|>a$. We define $F_{a}$ and $F_{b}$ as in (1.4). To prove (2.1) and (2.2), it is sufficient to prove that

$$
F_{a}(x)=F_{b}(x)-\pi^{-1} \int_{-a}^{a} \Gamma_{a}(x, u ; \lambda)\left\{\int_{a<|z|<b} \frac{F_{b}(z)}{(z-u)^{2}} d z\right\} d u
$$

for each $f \in C[-b,+b]$ and $|x|<a$.

Now, defining $\Omega_{b}$ as in Definition 1.1, we have for each $\lambda>0$ and $x \in(-a$, a),

$$
\begin{aligned}
\lambda F_{b}(x)-\Omega_{b} F_{b}(x)= & f(x)=\lambda F_{b}(x)-\pi^{-1} P . \int_{-a}^{a} \frac{F_{b}^{\prime}(t)}{t-x} d t \\
& -\pi^{-1} \int_{a<|z|<b} \frac{F_{b}^{\prime}(t)}{t-x} d t,
\end{aligned}
$$

the second equality holding almost everywhere in $[-b,+b]$.

For convenience we introduce the notations,

$$
H(u)=\int_{a<|z|<b} \frac{F_{b}(z)}{(z-u)^{2}} d z
$$

and

$$
F^{*}(x)=\pi^{-1} \int_{-a}^{a} \Gamma_{a}(x, u ; \lambda) H(u) d u .
$$

Since $H \in U[-a,+a]$ (see Definition 1.2), we have from Theorem 1.2

$$
\lambda F^{*}(x)-\tilde{\Omega}_{a} F^{*}(x)=H(x),
$$

where $\widetilde{\Omega}_{a}$ is the operator of Definition 1.3.

We next show that (2.7) can be rewritten as

$$
\begin{aligned}
\lambda F^{*}(x)-\pi^{-1} P . \int_{-a}^{a} & \frac{d / d t F^{*}(t)}{t-x} d t \\
& \quad-F^{*}(a)(a-x)^{-1}-F^{*}(-a)(a+x)^{-1}=H(x) .
\end{aligned}
$$

First we prove that

$$
\left[d / d x F^{*}(x)\right]^{2}\left(a^{2}-x^{2}\right)^{1 / 2} \in L(-a,+a) ;
$$

this assures that $\left[F^{*}(x)\right]^{\prime} \in L(-a,+a)$ and an application of $[3$, Theorem $4.2]$ accomplishes the proof of (2.8). 
To prove (2.9), we note that, by the last statement in Theorem 1.1, $H(x)$ is of the form,

$$
H(x)=F_{b}(-a)(a+x)^{-1}+F_{b}(a)(a-x)^{-1}+H_{1}(x)
$$

where $H_{1} \in U[-a,+a]$ and $H_{1} \in L_{p}(-a,+a)$ for every $p>1$. Hence

$$
F^{*}(x)=F_{b}(-a) \xi_{a}^{+}(x)+F_{b}(a) \overline{\xi_{a}}(x)+\int_{-a}^{+a} \Gamma_{a}(x, y ; \lambda) H_{1}(y) d y .
$$

Now $\xi_{a}^{ \pm}$by Theorem 1.3 are in the domain of $\Omega_{a}$ and hence satisfy condition (2.9) (with $F^{*}$ replaced by $\xi_{a}^{ \pm}$). That the integral in (2.11) satisfies this condition follows easily from [3, (2.9), Theorem 1.2 and Lemma 3.1].

We have now proved (2.8). An integration by parts in the integral on the right-hand sîde of $(2.8)$ gives

$$
\lambda F^{*}(x)-\pi^{-1} P . \int_{-a}^{a} \frac{d / d t F^{*}(t)}{t-x} d t=\pi^{-1} \int_{a<|z|<b} \frac{F_{b}^{\prime}(z)}{z-x} d z .
$$

Next put

$$
\phi(x)=F_{b}(x)-F_{a}(x)-F^{*}(x) .
$$

It follows from the definition of $F_{b}$ and $F_{a}$ and $(2.9)$ that $\left[\phi^{\prime}(x)\right]^{2}\left(a^{2}-x^{2}\right)^{1 / 2}$ $\in L[-a,+a]$ and that

$$
\lambda \phi-\Omega_{a} \phi=0 .
$$

Finally from $[3$, Corollary 3.1$]$ we get

$$
\lim _{x \rightarrow \pm a} F^{*}( \pm a)=F_{b}( \pm a) .
$$

Since (1.3) holds, $\phi( \pm a)=0$. But we showed in [2] that the only solution of (2.14) under these boundary conditions is $\phi(x) \equiv 0$, which proves (2.3).

Gơrollary 2.1. If $P_{a}$ and $P_{b}$ are defined as in $\S 0$, then (0.14) holds for $b>a, x \in(-a, a)$ and $S a$ Borel subset of $(-\infty, \infty)$.

Proof. Denote the difference between the right and left sides of (0.14) by $Q(t, x, S)$. Let $f$ be an element of $C[-\infty, \infty]$ for which $f( \pm a)=f( \pm b)=0$, and form the function

$$
F(t, x)=\int_{-\infty}^{\infty} Q(t, x, d y) f(y) .
$$

We have shown in Theorem 2.1 that the Laplace transform of $F$ with respect to $t$ is identically 0 . Since for each $x \in(-a, a)$, the function $F$ is continuous for $t \geqq 0$, we conclude that $F(t, x)=0$ for $x \in(-a, a)$ and $t \geqq 0$. From this it follows that $Q(t, x, S)=0$, except perhaps when $S$ contains the points $\pm a$, $\pm b$. However, since for any $c>0, P_{c}(t, x, S)=0$ if $S$ is contained in the com- 
plement of $(-c, c)$, it can be checked directly that $(0.14)$ holds in this case also.

3. The relation between the Cauchy process and the absorbing barrier processes. Define

$$
\Gamma(x, y ; \lambda)=\int_{0}^{\infty} e^{-\lambda t} p(t, x, y) d t
$$

for $\lambda>0$ where

$$
p(t, x, y)=\frac{t}{(x-y)^{2}+t^{2}} .
$$

The main object of this section is to prove the following theorems:

Theorem 3.1. If $\Gamma_{a}(x, y ; \lambda)$ is defined as in Theorem 1.1 and $\Gamma(x, y ; \lambda)$ as in (3.1), then for each $a>0$

$$
\Gamma(x, y ; \lambda)=\Gamma_{a}(x, y ; \lambda)+\pi^{-1} \int_{-a}^{a} \Gamma_{a}(x, u ; \lambda)\left\{\int_{|z|>a} \frac{\Gamma(z, y ; \lambda)}{(z-u)^{2}} d z\right\} d u
$$

when $|x|<a,|y|<a$ and

$$
\Gamma(x, y ; \lambda)=\pi^{-1} \int_{-a}^{a} \Gamma_{a}(x, u ; \lambda)\left\{\int_{|z|>a} \frac{\Gamma(z, y ; \lambda)}{(z-u)^{2}} d z\right\} d u
$$

when $|x|<a,|y|>a$.

This theorem expresses relation (0.13) in Laplace transforms.

Our second main theorem is:

THEOREM 3.2. If

$$
F(x)=\int_{-\infty}^{\infty} \Gamma(x, y ; \lambda) f(y) d y
$$

for some $\lambda>0$ and some $f \in C[-\infty,+\infty]$, then

$$
F(x)=\lim _{b \rightarrow \infty} F_{b}(x)
$$

where $F_{b}$ is given in (1.4). The convergence is uniform in any finite interval.

We first take up the

Proof of Theorem 3.1. Defining $F(x)$ as in (3.5) it follows from [6] that for fixed $\lambda$, there exists a set of $f$ dense in $C[-\infty,+\infty]$ for which

$$
\lambda F(x)-\pi^{-1} P . \int_{-\infty}^{\infty} \frac{F^{\prime}(t)}{t-x} d t=f(x)
$$

almost everywhere. (This can also be shown easily by choosing $f(x)$ of the 
form $u(\alpha, x)$, harmonic in $\alpha$ and $x$ with $\int_{-\infty}^{\infty}[u(\alpha, x)]^{2} d x<\infty$.) Now $F \in C[-\infty$, $+\infty]$, and we can rewrite (3.7) as

$$
\lambda F(x)-\tilde{\Omega}_{a} F(x)=\pi^{-1} \int_{|z|>a} \frac{F(z)}{(z-x)^{2}} d z+f(x)
$$

for any $a>0$ and $\widetilde{\Omega}_{a}$ as in Definition 1.8 .

The function on the right of (3.8) is an element of $U[-a,+a]$. Thus by Theorem 1.2,

(3.9) $F(x)=\int_{-a}^{a} \Gamma_{a}(x, y ; \lambda) f(y) d y+\pi^{-1} \int_{-a}^{a} \Gamma_{a}(x, u ; \lambda)\left\{\int_{|z|>a} \frac{F(z)}{(z-u)^{2}} d z\right\} d u$ for every $a>0$, whenever $f$ belongs to our dense set for which (3.8) holds, and $|x|<a$.

Each side of (3.9) defines a bounded linear transformation from $C[-\infty$, $+\infty]$ tọ $C[-a,+a]$, and thus the identity holds for all $f \in C[-\infty,+\infty]$. This in turn implies that (3.3) and (3.4) hold.

Corollary 3.1. If $P$ and $P_{a}$ are defined as in $\$ 0$, then (0.13) holds.

Proof. The proof is similar to that of Corollary 2.1 .

To prove Theorem 3.2, we require a lemma:

Lemma 3.1. If $\xi_{a}$ is given by (1.15) then

$$
\lim _{a \rightarrow \infty} \xi_{a}(x)=0
$$

and the convergence is uniform in any finite interval.

Proof. By [2, formula (2.9) and Lemma 5.1], it follows that

$$
\int_{-a}^{a} \Gamma_{a}(x ; y ; \lambda) d y+\lambda \int_{-a}^{a} \Gamma_{a}(x, y ; \lambda)\left(a^{2}-y^{2}\right)^{1 / 2} d y=\left(a^{2}-x^{2}\right)^{1 / 2}
$$

Therefore

$$
\int_{-a}^{a} \Gamma_{a}(x, y ; \lambda) d y=\lambda^{-1}\left[1-\xi_{a}(x)\right] \geqq\left(a^{2}-x^{2}\right)^{1 / 2}(1+a \lambda)^{-1} .
$$

It was shown in [2] that

$$
0 \leqq \xi_{a}(x) \leqq 1
$$

Combining (3.12) and (3.13), we obtain

$$
1 \geqq 1-\xi_{a}(x) \geqq \lambda\left(a^{2}-x^{2}\right)^{1 / 2}(1+a \lambda)^{-1},
$$

which proves the lemma.

We now proceed to: 
Proof of Theorem 3.2. We proved in [2, Theorem 4.2], that

$$
\lambda\left|F_{b}(x)\right| \leqq \max _{x \in[-b,+b]}|f(x)| .
$$

Therefore by (2.3) and (1.15), it follows that for $b>a$ and $|x|<a$,

$$
\left|F_{b}(x)-F_{a}(x)\right| \leqq \lambda^{-1}\|f\| \xi_{a}(x) .
$$

Thus by Lemma 3.1 the sequence $\left\{F_{b}(x)\right\}$ converges uniformly in any finite interval to a limit function $\Phi(x)$. We have now only to show that $\Phi$ coincides with the function $F$ defined by (3.5). Since the convergence of $F_{b}$ to $\Phi$ is bounded, we conclude from (2.3) that (3.9) holds with $F$ replaced by $\Phi$. Hence $F-\Phi=\Psi$ is a bounded solution of

$$
\Psi(x)=\pi^{-1} \int_{-a}^{a} \Gamma_{a}(x, u ; \lambda)\left\{\int_{|z|>a} \frac{\Psi(z)}{(z-u)^{2}} d z\right\} d u,
$$

for each $a>0$. But this implies that

$$
|\Psi(x)| \leqq M \xi_{a}(x) \text { for }|x|<a,
$$

and by Lemma 3.1 we must have $\Psi(x) \equiv 0$. This completes the proof of the theorem.

As a by-product of the investigations of this section, we determine the infinitesimal generator of the semi-group $\left\{T_{t}\right\}$ from $C[-\infty,+\infty]$ to itself defined by $(0.1)$.

Definition 3.1. We define $\Omega F=G$ if and only if (i) $F \in C[-\infty,+\infty]$ and $F$ is absolutely continuous over any finite interval; (ii) $F^{\prime}$ is square integrable over any finite interval (iii) $G \in C[-\infty,+\infty]$ and

$$
G(x)=\lim _{\alpha \rightarrow-\infty, \beta \rightarrow \infty} \pi^{-1} P \cdot \int_{\alpha}^{\beta} \frac{F^{\prime}(t)}{t-x} d t
$$

almost everywhere.

TheOREM 3.3. The operator $\Omega$ defined in Definition 3.1 is the infinitesimal generator of the semi-group $\left\{T_{t}\right\}$ from $C[-\infty,+\infty]$ to itself defined by (0.1).

Proof. The transformation $f \rightarrow F$ defined by (3.5) is the resolvent of this semi-group. To prove our theorem we need only show that (3.5) holds if and only if $\lambda F-\Omega F=f$, where $f \in C[-\infty,+\infty]$ and $\lambda>0$. First, suppose that $F$ is defined by (3.5). Then from Theorem 3.1 we have (3.9) for every $a>0$. Thus, $F(x)$ is of the form (1.4) with $f \in U[-a,+a]$ (the $f$ in (1.4) is here to be replaced by $f+\pi^{-1} \int_{|z|>a} F(z)(z-x)^{-2} d z$ in the notation of this theorem); by Theorem 1.2, it follows that $F$ also satisfies (3.8) and conditions (i) and (ii) of Definition 3.1. Since $F$ is absolutely continuous over any finite interval, it follows from [3, Theorem 4.2] that we may rewrite (3.8) as 


$$
\begin{array}{r}
\lambda F(x)-\pi^{-1} P \cdot \int_{-a}^{a} \frac{F^{\prime}(t)}{t-x} d t+\pi^{-1}\left[F(a)(a-x)^{-1}+F(-a)(a+x)^{-1}\right] \\
=f(x)+\pi^{-1} \int_{|z|>a} \frac{F(z)}{(z-x)^{2}} d z,
\end{array}
$$

almost everywhere. An integration by parts on the right of (3.19) over $(\alpha,-a)$ and $(a, \beta)$ gives $\lambda F-\Omega F=f$.

Conversely if $F \in$ domain $\Omega$, then define $f=\lambda F-\Omega F$. Clearly $F \in$ domain $\tilde{\Omega}_{a}$ for each $a>0$, and we may retrace our steps to obtain (3.19), (3.8) and finally (3.9). Since $F$ satisfies (3.9) for every $a>0$, it must coincide with (3.5), since we proved in the proof of Theorem 3.2 that (3.17) has no bounded solutions.

4. The integral equation of return processes. We now turn to the processes described in $\S(c)$ of the Introduction. We thus consider a fixed finite interval $(-a, a)$ with return probabilities $\tau(z) p(z, S)$ defined for $|z|>a$.

Here for fixed $z$ (where $|z|>a$ ) the function $p(z, S)$ is a probability measure on the Borel sets of the open interval $(-a, a)$. For fixed $S$ it is Borel-measurable in $z$. Finally $\tau(z)$ is a Borel-measurable function, $0 \leqq \tau(z) \leqq 1$. We complete the definition of $p$ by putting $p(z,\{a\})=p(z,\{-a\})=0$.

Let $\mathfrak{B}_{a}(t, x, S)$ denote the transition probabilities associated with this process, and put

$$
\Pi_{a}(x, S ; \lambda)=\int_{0}^{\infty} e^{-\lambda t} \mathfrak{B}_{a}(t, x, S) d t .
$$

An argument similar to that used in $\S(b)$ of the Introduction to obtain $(0.13)$ and (0.14) leads to the following

Integral equation for $\Pi_{a}$.

$$
\begin{aligned}
\Pi_{a}(x, S ; \lambda)=\gamma_{a}(x, S ; \lambda)+\pi^{-1} \int_{-a}^{a} \Gamma_{a}(x, u ; \lambda) \\
\cdot\left\{\int_{|z|>a} \frac{\tau(z)}{(z-u)^{2}}\left[\int_{-a}^{a} p(z, d v) \Pi_{a}(v, S ; \lambda)\right] d z\right\} d u .
\end{aligned}
$$

Here $\gamma_{a}$ is defined in (1.5); the interval of integration is open, but may be equally taken as closed provided one puts

$$
p(z,\{a\})=p(z,\{-a\})=0 .
$$

In this section we show that there exists a minimal solution to (4.2) which enjoys all properties required of Laplace transforms of transition probabilities. However, there may be additional admissible solutions of (4.2) and to prove uniqueness we introduce the following

Condition A. We say that condition $A$ is satisfied if there exist numbers 
$q<1$ and $0<\epsilon<a$ such that

$$
\tau(z) p\left(z, T_{\epsilon}\right)<q<1
$$

where

$$
T_{\epsilon}=(-a,-a+\epsilon) \cup(a-\epsilon, a) .
$$

We now prove

THEOREM 4.1. Define the iterated kernels $K_{n}$ inductively as follows:

$$
\begin{aligned}
K_{0}(x, S ; \lambda) & =\pi^{-1} \int_{-a}^{a} \Gamma_{a}(x, u ; \lambda)\left\{\int_{|z|>a} \frac{\tau(z)}{(z-u)^{2}} p(z, S) d z\right\} d u, \\
K_{n+1}(x, S ; \lambda) & =\int_{-a}^{a} K_{0}(x, d v ; \lambda) K_{n}(v, S ; \lambda) \\
& =\int_{-a}^{a} K_{n}(x, d v ; \lambda) K_{0}(v, S ; \lambda), \\
K_{n}(x,\{a\} ; \lambda) & =K_{n}(x,\{-a\} ; \lambda)=0 .
\end{aligned}
$$

Then

$$
\Pi_{a}(x, S ; \lambda)=\gamma_{a}(x, S ; \lambda)+\sum_{n=0}^{\infty} \int_{-a}^{a} K_{n}(x, d v ; \lambda) \gamma_{a}(v, S ; \lambda)
$$

is a minimal solution of the integral equation (4.2) in the following sense.

For each $x \in E$ this $\Pi_{a}$ is a completely additive function on the Borel sets $S$ of $E$, and

$$
0 \leqq \Pi_{a}(x, S ; \lambda) \leqq \lambda^{-1} .
$$

Every other non-negative solution $\Pi_{a}^{\prime}$ of (4.2) satisfies the inequality

$$
\Pi_{a}^{\prime} \geqq \Pi_{a} .
$$

If the condition A is satisfied, then (4.7) is the only solution of (4.2) which is bounded in $x$ and $S$ for each fixed $\lambda>0$. Furthermore, in this case the series in (4.7) converges uniformly in $x$ and $S$.

Finally, if condition A is satisfied and $\tau(z)=1$ for each $|z|>a$, then

$$
\Pi_{a}(x, E ; \lambda)=\lambda^{-1} .
$$

Note. By definition

$$
\Pi_{a}(x,\{a\} ; \lambda)=\Pi_{a}(x,\{-a\} ; \lambda)=0 .
$$

That $\Pi_{a}$ actually defines transition probabilities is an easy consequence of the Hille-Yosida theory and will be shown in $\S 5$ (at least under a slight additional condition). 
Proof. Let

$$
\Pi_{N, a}(x, S ; \lambda)=\gamma_{a}(x, S ; \lambda)+\sum_{n=0}^{N} \int_{-a}^{a} K_{n}(x, d v ; \lambda) \gamma_{a}(v, S ; \lambda) .
$$

Clearly, for each $v \in E$ and $S$

$$
0 \leqq \Pi_{N, a}(v, S ; \lambda) \leqq \Pi_{N+1, a}(v, S ; \lambda),
$$

$$
\Pi_{N+1, a}(x, S ; \lambda)=\gamma_{a}(x, S ; \lambda)+\int_{-a}^{a} K_{0}(x, d v ; \lambda) \Pi_{N, a}(v, S ; \lambda) .
$$

To prove that (4.8) holds we first note that by (1.5) and (1.15)

$$
\begin{aligned}
0 & \leqq \Pi_{N, a}(x, S ; \lambda) \leqq \Pi_{N, a}(x, E ; \lambda) \\
& =\lambda^{-1}\left\{1-\xi_{a}(x)\right\}+\lambda^{-1} \sum_{n=0}^{N} \int_{-a}^{a} K_{n}(x, d v ; \lambda)\left\{1-\xi_{a}(v)\right\} .
\end{aligned}
$$

Now, since $0 \leqq \tau(z) p(z, S) \leqq 1$, we have

$$
0 \leqq K_{0}(x, S ; \lambda) \leqq \xi_{a}(x) \leqq 1
$$

by (1.15) and (3.13). Therefore

$$
\sum_{n=0}^{N} \int_{-a}^{a} K_{n}(x, d v ; \lambda)\left\{1-\xi_{a}(v)\right\} \leqq \sum_{n=0}^{N}\left\{K_{n}(x, E ; \lambda)-K_{n+1}(x, E ; \lambda)\right\} .
$$

Combining the last three relations we have

$$
0 \leqq \Pi_{N, a}(x, S ; \lambda) \leqq \lambda^{-1}\left\{1-\xi_{a}(x)+K_{0}(x, E ; \lambda)-K_{N+1}(x, E ; \lambda)\right\} \leqq \lambda^{-1} .
$$

We conclude from (4.13) and (4.18) that for each fixed $x$ and $S$ the sequence $\Pi_{N, a}(x, S ; \lambda)$ converges; it follows from monotonic convergence that the limit function (4.7) is a solution of (4.2) and satisfies (4.8). This proves the first part of the theorem.

For any non-negative solution of (4.2) we have obviously

$$
\begin{aligned}
\Pi_{a}(x, S ; \lambda)= & \gamma_{a}(x, S ; \lambda)+\sum_{n=0}^{N} \int_{-a}^{a} K_{n}(x, d v ; \lambda) \gamma_{a}(v, S ; \lambda) \\
& +\int_{-a}^{a} K_{N+1}(x, d v ; \lambda) \Pi_{a}(v, S ; \lambda) .
\end{aligned}
$$

The minimality assertion (4.9) is a direct consequence of this. Also, it follows from (4.19) that $\Pi_{a}$ as defined by (4.7) is the unique bounded solution whenever

$$
\lim _{N \rightarrow \infty} K_{N}(x, E ; \lambda)=0 \text { for each } x .
$$

Suppose now that condition A is satisfied. From (4.6) and (4.16) we get 


$$
K_{N}(x, E ; \lambda) \leqq \int_{-a}^{a} K_{N-2}(x, d w ; \lambda)\left\{\int_{-a}^{a} K_{0}(w, d v ; \lambda) \xi_{a}(v)\right\}
$$

Choose $\epsilon$ so that (4.4) holds, and put for this fixed $\epsilon$

$$
\eta=\sup _{x \in E-T_{\epsilon}} \xi_{a}(x) .
$$

It follows from (3.12) that $0<\eta<1$. Clearly

$$
\int_{-a}^{a} K_{0}(w, d v ; \lambda) \xi_{a}(v) \leqq(1-\eta) K_{0}\left(w, T_{\epsilon} ; \lambda\right)+\eta K_{0}(w, E ; \lambda) .
$$

From the first equation in (4.6) we get using condition $A$ and (1.15)

$$
K_{0}\left(w, T_{\epsilon} ; \lambda\right) \leqq q \cdot \xi_{a}(w) \leqq q,
$$

and so by (4.23)

$$
\int_{-a}^{a} K_{0}(w, d v ; \lambda) \xi_{a}(v) \leqq q(1-\eta)+\eta=\alpha<1 .
$$

From (4.21) we see that

$$
K_{N}(x, E ; \lambda) \leqq \alpha K_{N-2}(x, E ; \lambda)
$$

which proves that (4.20) holds and that the series in (4.7) converges uniformly.

Finally, if $\tau(z)=1$ for each $|z|>a$, then the equality sign holds in (4.17) and this together with (4.20) implies (4.10). This completes the proof.

We conclude this section by an example which shows that in the absence of condition A the solution $\Pi_{a}$ of (4.7) need not be the only bounded solution of the integral equation (4.2).

If this example is modified by defining $\tau(z) \equiv 1$ for $|z| \geqq a$, then the new solution shows that even if $\tau(z)=1$ for each $|z|>a$ the minimal solution $\Pi_{a}$ does not necessarily satisfy (4.10). The probabilistic implication of this is described in the Introduction.

EXAmple. We first choose two sequences $\left\{\epsilon_{k}\right\}$ and $\left\{\eta_{k}\right\}$ of positive numbers for which

$$
\sum_{k=0}^{\infty} \epsilon_{k}<\infty, \quad \epsilon_{k} \downarrow 0, \quad \eta_{k} \downarrow 0 .
$$

Let

$$
\begin{aligned}
& A_{0}=\left\{x \in[-a, a] / \xi_{a}(x)>1-\epsilon_{0}\right\}, \\
& A_{k}=\left\{x \in[-a, a] / \pi^{-1} \int_{-a}^{a} \Gamma_{a}(x, u ; \lambda)\left[\int_{a}^{a+\eta_{k}} \frac{d z}{(z-u)^{2}}\right] d u>1-\epsilon_{k}\right\} .
\end{aligned}
$$

Each $A_{k}$ contains an interval $[a-\delta, a]$ since, for each $\eta>0$, 


$$
\lim _{x \rightarrow \pm a}\left\{\pi^{-1} \int_{-a}^{a} \Gamma_{a}(x, u ; \lambda)\left[\int_{a}^{a+\eta} \frac{d z}{(z-u)^{2}}\right] d u\right\}=1
$$

by [3, Corollary 3.1]. It is also clear that $A_{k+1} \subset A_{k}$.

We now choose $\tau(z)$ and $p(z, S)$ to satisfy the following conditions:

$$
\begin{array}{rlr}
\tau(z) & \equiv 0, & z \leqq-a, \\
\tau(z) & \equiv 1, & z \geqq a, \\
p\left(z, A_{k+1}\right) & =1, & a<z<a+\eta_{k} .
\end{array}
$$

The sets $A_{k}$ contract as $k \rightarrow \infty$ to the end points $\pm a$, and so condition $\mathrm{A}$ is violated.

A bounded solution to the equation

$$
F(x, S)=\int_{-a}^{a} F(v, S) K_{0}(x, d v ; \lambda)
$$

may be constructed by successive approximation as follows: Put

By (4.16)

$$
\begin{aligned}
F_{0}(x, S) & \equiv 1 \\
F_{n+1}(x, S) & =\int_{-a}^{a} K_{0}(x, d v ; \lambda) F_{n}(v, S) .
\end{aligned}
$$

and by induction

$$
0 \leqq F_{1}(x, S) \leqq 1=F_{0}(x, S)
$$

Thus

$$
0 \leqq F_{n+1}(x, S) \leqq F_{n}(x, S) \leqq 1
$$

exists for each fixed $x$ and $S$ and satisfies (4.31).

We have only to show that $F(x, S) \neq 0$. First,

$$
\begin{aligned}
& F_{n}(x, S) \geqq \int_{-a}^{a} \Gamma_{a}(x, u ; \lambda)\left\{\int_{a}^{a+\eta k}(z-u)^{-2} \int_{A_{k+1}} p(z, d v) F_{n-1}(v, S)\right\} d u \\
& \geqq\left\{\min _{x \in A_{k+1}} F_{n-1}(x, S)\right\}\left\{\int_{-a}^{+a} \Gamma_{a}(x, u ; \lambda)\left[\int_{a}^{a+\eta k} \frac{d z}{(z-u)^{2}}\right] d u\right\} .
\end{aligned}
$$

Therefore, for $x \in A_{k}$,

$$
F_{n}(x, S) \geqq\left(1-\epsilon_{k}\right) \min _{x \in A_{k+1}} F_{n-1}(x, S) .
$$

Now for $x \in A_{0}$, 


$$
\begin{aligned}
F_{n}(x, S) & \geqq\left(1-\epsilon_{0}\right) \min _{x \in A_{1}} F_{n-1}(x, S) \\
& \geqq \prod_{k=0}^{n-1}\left(1-\epsilon_{k}\right) \min _{x \in A_{n}} F_{0}(x, S) \\
& =\prod_{k=0}^{n-1}\left(1-\epsilon_{k}\right),
\end{aligned}
$$

by repeated application of (4.37). Hence, for $x \in A_{0}$,

$$
F(x, S) \geqq \prod_{k=0}^{\infty}\left(1-\epsilon_{k}\right)>0 .
$$

But $A_{0}$ is a set of positive measure, and thus $F$ is a nontrivial bounded solution of (4.31).

5. The associated semi-group. Let $\Pi_{a}(x, S ; \lambda)$ be the solution (4.7) of the integral equation (4.2). For $f \in C[-a, a]$ put

$$
R_{\lambda} f(x)=\int_{-a}^{a} \Pi_{a}(x, d v ; \lambda) f(v), \quad x \in E .
$$

We introduce a condition on the function $p$ which assures that (5.1) defines a bounded linear transformation from $C[-a, a]$ to itself.

Condition B. For each $f \in C[-a, a]$

$$
\underset{z \rightarrow \pm a}{\operatorname{essential~limit}} \tau(z) \int_{-a}^{a} p(z, d v) f(v)
$$

exists.

The two main theorems of this section are:

TheOREM 5.1. Let $\Pi_{a}$ be defined by (4.7); under conditions $\mathrm{A}$ and $\mathrm{B}$ the transformation $R_{\mathrm{\lambda}}$ defined by (5.1) is a bounded linear transformation from $C[-a,+a]$ to itself and

$$
\left\|R_{\lambda}\right\| \leqq \lambda^{-1} \text {. }
$$

If $\tau(z) \equiv 1$, then equality holds in (5.3). (See $\$ 4$ for definition of $\tau$.)

THEOREM 5.2. Under conditions $\mathrm{A}$ and $\mathrm{B}$, if $F=R_{\lambda}$ for some $f \in C[-a,+a]$ and some $\lambda<0$, then $F$ is the unique solution in $C[-a,+a]$ of

$$
\lambda F(x)-\tilde{\Omega}_{a} F(x)-\int_{|z|>a} \frac{\tau(z) \int_{-a}^{a} p(z, d v) F(v)}{(z-x)^{2}}=f(x)
$$

where $p$ and $\tau$ are defined in $\S 4$ and $\widetilde{\Omega}_{a}$ is the operator of Definition 1.3. 
Define

$$
A F(x)=\tilde{\Omega}_{a} F(x)+\int_{|z|>a} \frac{\tau(z) \int_{-a}^{a} p(z, d v) F(v)}{(z-x)^{2}} d z
$$

and let $D(A)$ denote the set of $F \in C[-a,+a]$ for which the right side of (5.5) is in $C[-a,+a]$. Thus $A$ defines a transformation on $C[-a,+a]$ to itself with domain $D(A)$. Theorem 5.2 shows that for each $\lambda>0$, the range of $R_{\lambda}$ coincides with $D(A)$. From the Hille-Yosida theorem and Theorems 5.1 and 5.2 we conclude that $\mathrm{A}$ is the infinitesimal generator of a semi-group $\left\{U_{t}\right\}$ from $\overline{\mathscr{D}(A)}$ to itself, which is strongly continuous for $t \geqq 0$. This semigroup can be written in the form

$$
U_{t} f(x)=\int_{[-a,+a]} \mathfrak{P}_{a}(t, x, d v) f(v) .
$$

Since $U_{t}$ is positivity preserving and $\left\|U_{t}\right\| \leqq 1$ (again by the Hille-Yosida theorem), we see that for each $x, \mathfrak{B}_{a}(t, x, S)$ defines a probability measure on the Borel subsets of $[-a,+a]$. The function $\mathfrak{P}$ gives the transition probabilities of the return process of $\S(\mathrm{c})$ in the Introduction.

The backward equation for the return process is thus given by

$$
\frac{\partial}{\partial t} U_{t} f(x)=A U_{t} f(x)
$$

and if $f \in \overline{\mathscr{D}(A)}$, then $\lim _{t \rightarrow 0} U_{t} f(x)=f(x)$ uniformly in $x$.

We shall also show, under our conditions $\mathrm{A}$ and $\mathrm{B}$, that $\overline{\mathrm{D}(A)}$ is actually a proper subspace of $C[-a,+a]$ :

THEOREM 5.3. Under conditions $\mathrm{A}$ and $\mathrm{B}$ the range of $R_{\lambda}$ is contained in the subspace of $C[-a,+a]$ of functions $F$ satisfying

$$
F( \pm a)=\underset{z \rightarrow \pm a}{\operatorname{ess} .} \lim \tau(z) \int_{E} p(z, d v) F(v) .
$$

The set defined by (5.8) is a closed linear manifold in $C[-a,+a]$. Clearly, under condition $\mathrm{A}$, this manifold cannot coincide with $C_{1}[-a, a]$.

We now proceed to prove the theorems of this section.

Proof of Theorem 5.1. From (4.7), we have for each $f \in C[-a,+a]$,

$$
\begin{aligned}
R_{\lambda} f(x)= & \int_{-a}^{a} \Gamma_{a}(x, u ; \lambda) f(u) d u \\
& +\sum_{n=0}^{\infty} \int_{-a}^{a} f(u)\left\{\int_{-a}^{a} \Gamma_{a}(v, u ; \lambda) K_{n}(x, d v ; \lambda)\right\} d u,
\end{aligned}
$$


where the series on the right converges uniformly in $x$. Let us consider the first term of the series in (5.9),

$$
\begin{aligned}
S_{0}(x) & =\int_{-a}^{a} K_{0}(x, d v ; \lambda)\left\{\int_{-a}^{a} \Gamma_{a}(v, u ; \lambda) f(u) d u\right\} \\
& =\int_{-a}^{a} K_{0}(x, d v ; \lambda) F_{a}(v) .
\end{aligned}
$$

Now $F_{a}(v) \in C[-a,+a]$, and it is easily seen by the definition of $K_{0}$ and condition B that $S_{0}$ is of the form (1.4) with $f \in U[-a,+a]$. Hence $S_{0} \in C[-a$ $+a]$. Letting $S_{n}$ represent the $n$th term of the series we have

$$
S_{n+1}(x)=\int_{-a}^{a} K_{0}(x, d v ; \lambda) S_{n}(v)
$$

and it follows by induction that $S_{n} \in C[-a,+a]$ for every $n$. Thus, since the series $\sum_{n=0}^{\infty} S_{n}(x)$ converges uniformly in $x$, we conclude that $R_{\lambda} f \in C[-a$, $+a$ ]. Finally (5.3) follows from (4.8).

Proof of Theorem 5.2. It is now very simple to prove Theorem 5.2. If $F=R_{\lambda} f$ for some $f \in C[-a,+a]$, then from (4.2) we have

$$
\begin{aligned}
F(x) & =F_{a}(x) \\
& +\pi^{-1} \int_{-a}^{a} \Gamma_{a}(x, u ; \lambda)\left\{\int_{|z|>a} \frac{\tau(z)}{(z-u)^{2}}\left[\int_{-a}^{a} p(z, d v) F(v)\right] d z\right\} d u
\end{aligned}
$$

with $F_{a}$ given by (1.4). Since $F \in C[-1,+1]$, it follows from condition B that the function in braces in (5.12) is an element of $U[-a,+a]$. Hence (5.4) follows from Theorem 1.2. The uniqueness follows from (4.20).

Proof of Theorem 5.3. We showed in [3, Corollary 3.1], that if $F_{a}$ is given by (1.4) with $f \in U[-a,+a]$, then

$$
\lim _{x \rightarrow \pm a} F(x)=(2 a)^{-1} \cdot \pi \lim _{x \rightarrow \pm a} f(x)\left(a^{2}-x^{2}\right) .
$$

From this it is easily seen that (5.8) holds.

6. The adjoint semi-group. In this section we shall consider the transformation

$$
R_{\lambda}^{*} \mu(S)=\int_{-a}^{+a} \Pi_{a}(x, S ; \lambda) \mu(d x)
$$

where $\mu$ is a measure on the open interval $(-a,+a)$ and $\Pi_{a}$ is defined by (4.7). We shall prove the following two theorems:

THEOREM 6.1. Under conditions A and $\mathrm{B}$, the transformation $R_{\lambda}^{*}$ given by (6.1) is a bounded linear transformation from the space $\mathfrak{T C}$ of totally finite measures on the open interval $(-a,+a)$ to itself, for each $\lambda>0$, and 


$$
\left\|R_{\lambda}^{*} \mu\right\| \leqq \bar{\lambda}^{-1}\|\mu\| .
$$

Furthermore, $R_{\lambda}^{*} \mu$ is an absolutely continuous measure for which

$$
\int_{-a}^{+a} \frac{R_{\lambda}^{*} \mu(d x)}{a^{2}-x^{2}}<\infty
$$

and

THEOREM 6.2. If $M=R_{\lambda}^{*} \mu$ for some $\lambda>0$ and some $\mu \in \mathscr{T}$, then under conditions $\mathrm{A}$ and $\mathrm{B}$ we have for almost all intervals $I \subset(-a,+a)$,

$$
\lambda M(I)-A^{*} M(I)=\mu(I)
$$

where

(6.5) $A^{*} M(I)=\pi^{-1} \int_{I} \frac{d}{d x} P . \int_{-a}^{a} \frac{M(d y)}{y-x}+\int_{-a}^{a} M(d u) \int_{|z|>a} \frac{\tau(z) p(z, I)}{(z-u)^{2}} d z$.

These two theorems show that $R_{\lambda}^{*}$ is the resolvent of a semi-group $\left\{U_{t}^{*}\right\}$ from $\mathfrak{T}$ to itself, whose infinitesimal generator is of the form (6.5). Thus the "forward equation" for our return processes can be written as

$$
\frac{\partial}{\partial t} U_{t} \mu(I)=A^{*} U_{t} \mu(I)
$$

Proof of Theorem 6.1. Consider the function

$$
\Psi(S)=\sum_{n=0}^{\infty} \int_{-a}^{a} K_{n}(x, S ; \lambda) \mu(d x)
$$

for a fixed measure $\mu$ on $(-a,+a)$. Here $\Psi$ is defined on Borel subsets $S$ of $(-a,+a)$. From (4.26) we conclude that $\Psi$ is a non-negative, totally finite measure on $(-a,+a)$. Moreover,

$$
M(S)=R_{\lambda}^{*} \mu(S)=\int_{-a}^{a} \Psi(d v) \gamma_{a}(v, S ; \lambda)+\int_{-a}^{a} \mu(d x) \gamma_{a}(x, S ; \lambda) .
$$

By Lemma 11.1 in [2] it follows that $M$ is an absolutely continuous measure. To obtain (6.3) we note that

$$
\begin{aligned}
\int_{-a}^{a} \frac{M(d x)}{a^{2}-x^{2}} & =\int_{-a}^{a}\{\Psi(d v)+\mu(d v)\} \int_{-a}^{a} \frac{\Gamma_{a}(v, x ; \lambda)}{\left(a^{2}-x^{2}\right)} d x \\
& \leqq\{\|\Psi\|+\|\mu\|\} \cdot \pi(2 a)^{-1}
\end{aligned}
$$

by (1.15) and (3.13). This completes the proof.

Proof of Theorem 6.2. Using (6.7) and Lemma 11.1 in [2], we have 


$$
M(I)-\pi^{-1} \int_{I}\left\{\frac{d}{d x} P \cdot \int_{-a}^{a} \frac{M(d y)}{y-x}\right\} d x=\Psi(I)+\mu(I),
$$

for almost all intervals $I$. To obtain (6.4), we shall show that

$$
\Psi(I)=\int_{-a}^{a} M(d u)\left\{\int_{|z|>a} \frac{\tau(z) p(z, I)}{(z-u)^{2}} d z\right\} .
$$

Note that the integral on the right of (6.10) exists by (6.3). It is easily verified that

$$
\Psi(I)=\int_{-a}^{a} \Psi(d v) K_{0}(v, I ; \lambda)+\int_{-a}^{a} \mu(d v) K_{0}(v, I ; \lambda) .
$$

On the other hand, letting

$$
L(u, S)=\int_{|z|>a} \frac{\tau(z) p(z, S)}{(z-u)^{2}} d z
$$

we have

$$
\begin{aligned}
\int_{-a}^{a} \Psi(d v) K_{0}(v, I ; \lambda) & =\int_{-a}^{a} \Psi(d v) \int_{-a}^{a} \gamma_{a}(v, d u ; \lambda) L(u, I) \\
& =\int_{-a}^{a} L(u, I) \int_{-a}^{a} \Psi(d v) \gamma_{a}(v, d u ; \lambda) \\
& =\int_{-a}^{a} L(u, I)\left\{M(d u)-\int_{-a}^{a} \gamma_{a}(x, d u ; \lambda) \mu(d x)\right\}
\end{aligned}
$$

by (6.7). This completes the proof.

7. Return processes leading to harmonic functions. In this section we deal with return processes of the type described in $\S(\mathrm{d})$ of the Introduction.

We first take up a return process which leads to the semi-group (0.26). For simplicity we shall consider the process on $(-1,+1)$. In this case $\tau(z) \equiv 1$ and

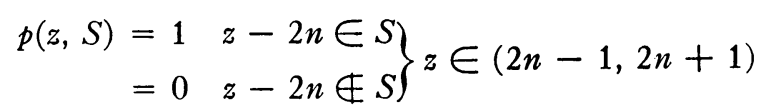

where $S$ is a Borel subset of $(-1,1)$. Note that condition A is not satisfied for this choice of $\tau$ and $p$. Using the periodicity of $p$, we may write the integral equation (4.2) as

$$
\begin{aligned}
\Pi_{1}(x, S ; \lambda)= & \gamma_{1}(x, S ; \lambda) \\
& +\pi^{-1} \int_{-1}^{1} \Gamma_{1}(x, u ; \lambda)\left\{\int_{-1}^{1} \Pi_{1}(z, S ; \lambda) R(z, u) d z\right\} d u,
\end{aligned}
$$


where

$$
R(z, u)=\sum_{n \neq 0} \frac{1}{(z-2 n-u)^{2}}=\frac{\pi^{2}}{4}\left[\sin \frac{\pi}{2}(z-u)\right]^{-2}-(z-u)^{-2} .
$$

It is easily seen from (3.3) and (3.4) that a solution of (7.2) is given by

$$
\Pi_{1}(x, S ; \lambda)=\int \sum_{S} \sum_{k=-\infty}^{+\infty} \Gamma(x, y+2 k ; \lambda)
$$

for $\lambda>0$. However, since condition $A$ is violated we cannot use the argument of Theorem 4.1 to conclude that (7.4) is the only admissible solution. The next theorem establishes this uniqueness (up to sets of measure zero in $x$ ).

THEOREM 7.1. For each $\lambda>0$, the function $\Pi_{1}$ defined by (7.4) is the only solution of (7.2) for which $0 \leqq \Pi_{1}(x, S ; \lambda) \leqq M<\infty$ with $M$ independent of $x$ and $S$.

Proof. Define a linear transformation $T$ by

$$
T \phi(x)=\pi^{-1} \int_{-1}^{1} \Gamma_{1}(x, u ; \lambda)\left\{\int_{-1}^{1} \phi(z) R(z, u) d z\right\} d u .
$$

If there existed two bounded solutions to (7.2), then their difference would be, for each fixed $S$, a bounded solution of

$$
T \phi(x)=\phi(x) .
$$

We may assume $|\phi(x)| \leqq 1$; since $T$ is a positivity preserving transformation $|\phi(x)|=\left|T^{n} \phi(x)\right| \leqq T^{n} 1$, where 1 denotes the function identically equal to 1 in $[-1,+1]$. To show that (7.6) implies $\phi(x) \equiv 0$, it is therefore sufficient to show that $T^{n} 1 \rightarrow 0$ as $n \rightarrow \infty$ for each $x \in(-1,+1)$. Now

$$
0 \leqq T 1=\xi_{1} \leqq 1
$$

where $\xi_{1}$ is defined by (1.15). Hence,

$$
0 \leqq T^{n+1} 1 \leqq T^{n} 1 \leqq 1,
$$$$
n \geqq 1 \text {. }
$$

We conclude from this that $T^{n} 1(x)$ converges to a limit function $\psi \geqq 0$.

Moreover,

$$
\psi(x)=\pi^{-1} \int_{-1}^{1} \Gamma_{1}(x, u ; \lambda)\left\{\int_{-1}^{1} \psi(z) R(z, u) d z\right\} d u .
$$

Using (1.15), we have

$$
2 \int_{-1}^{1} \psi(x)\left(1-x^{2}\right)^{-1} d x=\int_{-1}^{1} \xi_{1}(u)\left\{\int_{-1}^{1} \psi(z) R(z, u) d z\right\} d u
$$


But

$$
\int_{-1}^{1} R(x, u) d u=2\left(1-x^{2}\right)^{-1}
$$

and (7.10) becomes

$$
\int_{-1}^{1} \psi(x)\left\{\int_{-1}^{1} R(x, u) d u\right\} d x=\int_{-1}^{1} \xi_{1}(u)\left\{\int_{-1}^{1} \psi(z) R(z, u) d z\right\} d u,
$$

and thus

$$
0=\int_{-1}^{1} \psi(z)\left\{\int_{-1}^{1} R(z, u)\left[1-\xi_{1}(u)\right] d u\right\} d z .
$$

But the function in braces is a positive function and $\psi(z) \geqq 0$. Hence, $\psi(z)=0$ almost everywhere.

THEOREM 7.2. If, for some $\lambda>0$ and some $f \in C[-1,+1]$,

$$
F(x)=\int_{-1}^{1} \Pi_{1}(x, d y ; \lambda) f(y),
$$

where $\mathrm{I}_{1}$ is given by (7.4), then

$$
\lambda F(x)-\frac{1}{2} P \cdot \int_{-1}^{1} F^{\prime}(t) \cot \frac{\pi}{2}(t-x) d t=f(x)
$$

almost everywhere on $[-1,+1]$.

Proof. By (7.2), $F$ satisfies

$$
\begin{aligned}
F(x)= & \int_{-1}^{1} \Gamma_{1}(x, u ; \lambda) f(u) d u \\
& +\pi^{-1} \int_{-1}^{1} \Gamma_{1}(x, u ; \lambda)\left\{\int_{-1}^{1} F(z) R(z, u) d z\right\} d u,
\end{aligned}
$$

and therefore is of the form (1.4) for some $f \in U[-a,+a]$. By Theorem 1.2,

$$
\lambda F(x)-\tilde{\Omega}_{1} F(x)=f(x)+\int_{-1}^{1} R(z, u) F(u) d u .
$$

By [3, Theorem 4.2], we can simplify this expression if $F$ is absolutely continuous on $(-1,+1)$. That this is so can be easily verified; let $f$ have the Fourier expansion

$$
f(x) \sim \sum_{n=0}^{\infty} a_{k} \cos k \pi x+b_{k} \sin k \pi x
$$


1956] STOCHASTIC PROCESSES CONNECTED WITH HARMONIC FUNCTIONS 419

then

$$
F(x)=\sum_{n=0}^{\infty}(\lambda+n \pi)^{-1}\left\{a_{n} \cos k \pi x+b_{k} \sin k \pi x\right\},
$$

the series converging uniformly. From (7.19) we see that $F$ is absolutely continuous on $(-1,+1)$. Hence [3, Theorem 4.2] we can rewrite (7.17) in the form

$$
\begin{aligned}
F(x)- & \pi^{-1} P \cdot \int_{-1}^{1} \frac{F^{\prime}(t)}{t-x} d t \\
& =f(x)+\pi^{-1} \int_{-1}^{1} F(z)\left\{\frac{\pi^{2}}{4}\left[\sin \frac{\pi}{2}(z-u)\right]^{-2}-(z-u)^{-2}\right\} d z \\
& -F(1)(1-x)^{-1}-F(-1)(1+x)^{-1} \\
& =f(x)+\pi^{-1} \int_{-1}^{1} F^{\prime}(z)\left\{\frac{\pi}{2} \cot \frac{\pi}{2}(z-u)-(z-u)^{-1}\right\} d z
\end{aligned}
$$

which, upon rearrangement gives (7.15).

It follows from (7.18) and (7.19) that the semi-group associated with this process has the representation

$$
T_{t} f=\sum_{n=0}^{\infty} e^{-n \pi t}\left\{a_{n} \cos n \pi x+b_{n} \sin n \pi x\right\} .
$$

The infinitesimal generator is

$$
\Omega F=\frac{1}{2} P \cdot \int_{-1}^{1} F^{\prime}(t) \cot \frac{\pi}{2}(t-x) d t
$$

where the domain of $\Omega$ consists of those $F$ which are absolutely continuous on $[-1,1]$, and for which the right side of (7.22) is equivalent to an element of $C[-1,+1]$.

A return process similar to the one described above is obtained by choosing $\tau(z) \equiv 1$ and

$$
\left.\begin{array}{rlrl}
p(z, S) & =1 & & (-1)^{n}(z-2 n) \in S \\
& =0 & & (-1)^{n}(z-2 n) \notin S
\end{array}\right\} z \in(2 n-1,2 n+1) .
$$

In this case the resolvent kernel satisfies,

$$
\begin{aligned}
& \Pi_{1}(x, S ; \lambda)=\gamma_{1}(x, S ; \lambda) \\
& +\pi^{-1} \int_{-1}^{1} \Gamma_{1}(x, u ; \lambda) d u \int_{-1}^{1} \Pi_{1}(z, S ; \lambda)\left\{\sum_{n \neq 0}\left[(-1)^{n_{z}}-u+2 n\right]^{-2}\right\} d z .
\end{aligned}
$$

Now 


$$
\begin{aligned}
\sum_{n \neq 0}\left[(-1)^{n} z-u+2 n\right]^{-2}= & \frac{\pi^{2}}{16}\left[\left\{\sin \frac{\pi}{4}(z-u)\right\}^{-2}\right. \\
& \left.+\left\{\cos \frac{\pi}{4}(z+u)\right\}^{-2}\right]-(z-u)^{-2}
\end{aligned}
$$

and if $F$ is defined by (7.14) we obtain as before

$$
\lambda F(x)-\frac{1}{4} P \cdot \int_{-1}^{1} F^{\prime}(t)\left\{\cot \frac{\pi}{4}(t-x)+\tan \frac{\pi}{4}(t+x)\right\} d t=f
$$

The semi-group on $C[-1,+1]$ associated with this process is

$$
T_{t} f(x)=\sum_{n=0}^{\infty} e^{-n \pi t / 2} a_{n} \cos \frac{n \pi}{2}(x-1)
$$

where $f$ has the formal expansion (7.27) with $t=0$. The infinitesimal generator is given by

$$
\Omega F=\frac{1}{4} P \cdot \int_{-1}^{1} F^{\prime}(t)\left\{\cot \frac{\pi}{4}(t-x)+\tan \frac{\pi}{4}(t+x)\right\} d t,
$$

the domain of $\Omega$ consisting of those $F \in C[-1,+1]$ which are absolutely continuous on $(-1,+1)$ and for which the right side of $(7.28)$ is equal almost everywhere to an element of $C[-1,+1]$.

\section{REFERENCES}

1. J. L. Doob, Markoff chains-denumerable case, Trans. Amer. Math. Soc. vol. 58 (1945) pp. $455-473$.

2. J. Elliott, The boundary value problems and semi-groups associated with certain integrodifferential operators, Trans. Amer. Math. Soc. vol. 76 no. 2 (1954) pp. 300-331.

3. - On an integro-differential operator of the Cauchy type, Proc. Amer. Math. Soc. vol. 7 (1956) pp. 616-626.

4. W. Feller, The parabolic differential equations and the associated semi-groups of transformations, Ann. of Math. vol. 55 (1952) pp. 468-519.

5. - Diffusion processes in one dimension, Trans. Amer. Math. Soc. vol. 77 no. 1 (1954) pp. 1-31.

6. E. Hille, On the generation of semi-groups and the theory of conjugate functions, Kungl. Fysiografiska Sällskapets i Lund Förhandlingar vol. 21 (1952).

7. M. Kac, On some connections between probability theory and differential and integral equations, Proceedings of the 2nd Symposium on Probability and Statistics, Berkeley, 1950, pp. 189215.

8. M. Kac and H. Pollard, The distribution of the maximum of partial sums of independent random variables, Canadian Journal of Mathematics vol. 2 (1950) pp. 375-384. $564-579$.

9. H. P. McKean, Jr., Sample functions of stable processes, Ann. of Math. vol. 61 (1955) pp.

Columbia University,

NEW YORK, N. Y.

Princeton UNIVERSity,

Princeton, N. J. 